\title{
The accuracy of linear indices of ventricular volume in pediatric hydrocephalus: technical note
}

\author{
Dustin K. Ragan, PhD, ${ }^{1}$ Jonathon Cerqua, BS, ${ }^{2}$ Tiffany Nash, BA, ${ }^{2}$ \\ Robert C. McKinstry, MD, PhD, ${ }^{3}$ Joshua S. Shimony, MD, PhD, ${ }^{3}$ Blaise V. Jones, MD, ${ }^{2}$ \\ Francesco T. Mangano, DO, ${ }^{4}$ Scott K. Holland, PhD, ${ }^{2}$ Weihong Yuan, PhD, ${ }^{2}$ \\ and David D. Limbrick Jr., MD, PhD'
}

\begin{abstract}
Departments of ${ }^{1}$ Neurosurgery and ${ }^{3}$ Radiology, Washington University School of Medicine, St. Louis, Missouri; and Departments of ${ }^{2}$ Radiology and ${ }^{4}$ Neurosurgery, University of Cincinnati, Ohio
\end{abstract}

\begin{abstract}
Assessment of ventricular size is essential in clinical management of hydrocephalus and other neurological disorders. At present, ventricular size is assessed using indices derived from the dimensions of the ventricles rather than the actual volumes. In a population of 22 children with congenital hydrocephalus and 22 controls, the authors evaluated the relationship between ventricular volume and linear indices in common use, such as the frontooccipital horn ratio, Evans' index, and the bicaudate index. Ventricular volume was measured on high-resolution anatomical MR images. The frontooccipital horn ratio was found to have a stronger correlation with both absolute and relative ventricular volume than other indices. Further analysis of the brain volumes found that congenital hydrocephalus produced a negligible decrease in the volume of the brain parenchyma.
\end{abstract}

http://thejns.org/doi/abs/10.3171/2014.10.PEDS14209

KEY WORDS hydrocephalus; MRI; volumes; linear indices

A SSESSMENT of cerebral ventricular size is essential in the clinical management of hydrocephalus and other neurological and neurosurgical disorders..$^{1,410}$ At present, ventricular size is most often estimated on 2D ultrasound, CT, or MR images using either a gestalt impression or linear indices such as Evans' index, frontal horn index, and bicaudate index. ${ }^{2,6,8,9}$ In recent years, the frontooccipital horn ratio (FOHR) has gained popularity, as it accounts to some degree for variations in ventricular configuration. ${ }^{8}$

The relationship between linear indices and ratios and true ventricular volume has been evaluated in a handful of studies using a variety of imaging modalities (CT, MRI, and ultrasound) and heterogeneous patient groups. Reports by O'Hayon et al. ${ }^{8}$ and Bourne et al. ${ }^{3}$ observed a correlation between FOHR and ventricular volume, with other measures having lower correlations between the index and ventricular volume.

The current study uses standardized, high-resolution MRI data acquired prospectively from a uniform cohort of pediatric patients with congenital hydrocephalus prior to treatment to examine the relationship between ventricular volumes and 2D indices of ventricular size. This study investigates the utility of FOHR in estimating ventricular size on 2D images where volumetric assessments may not be available due to technological limitations or clinical urgency.

\section{Methods}

\section{Subjects}

Twenty-two children (age range 0-195 months) diagnosed with congenital hydrocephalus and referred for surgical treatment were included in the study. Twenty-two age-matched children with normal findings on brain MRI (controls; age range 0-71 months) were also included. All participants were recruited for a multicenter study from Cincinnati Children's Hospital Medical Center and St. Louis Children's Hospital. The study protocol was approved by both local institutional review boards, and families of the participants gave written informed consent; children provided written assent if age appropriate. 


\section{Data Acquisition}

Subjects at Cincinnati Children's Hospital Medical Center underwent imaging in a 1.5-T Signa MRI scanner (GE Healthcare) with an SPGR (spoiled gradient-recalled acquisition) sequence (TR 11.9, TE 5, and TI $300 \mathrm{msec}$ ). Subjects at St. Louis Children's Hospital underwent imaging in a 1.5-T Avanto scanner (Siemens) with an MPRAGE (magnetization-prepared rapid acquisition gradient echo) sequence (TR 1900, TE 2.8, and TI $1100 \mathrm{msec}$ ). Images were acquired with FOV $256 \times 256 \times 176 \mathrm{~mm}$ and matrix size $256 \times 256 \times 176$. Details regarding data quality and consistency checks across institutions have been published elsewhere. ${ }^{11}$

\section{Data Analysis}

The following linear indices of ventricular size were determined: frontal horn index, FOHR, Evans' index, and the bicaudate index. ${ }^{2,6,8,9}$ The distance measures used to determine the indices were taken from manual distance measurements on the T1-weighted images (Fig. 1). For 20 of the subjects with hydrocephalus, occipitofrontal head circumferences were obtained from the patient's charts; the other 2 subjects did not have data available. Intracranial volume (ICV) and ventricular volume were determined using Vitrea (Philips Healthcare), which allows semiautomatic segmentation of tissues. Ventricular volumes included the lateral, third, and fourth ventricles. Two subjects were excluded from analysis due to significant motion or metal ar-

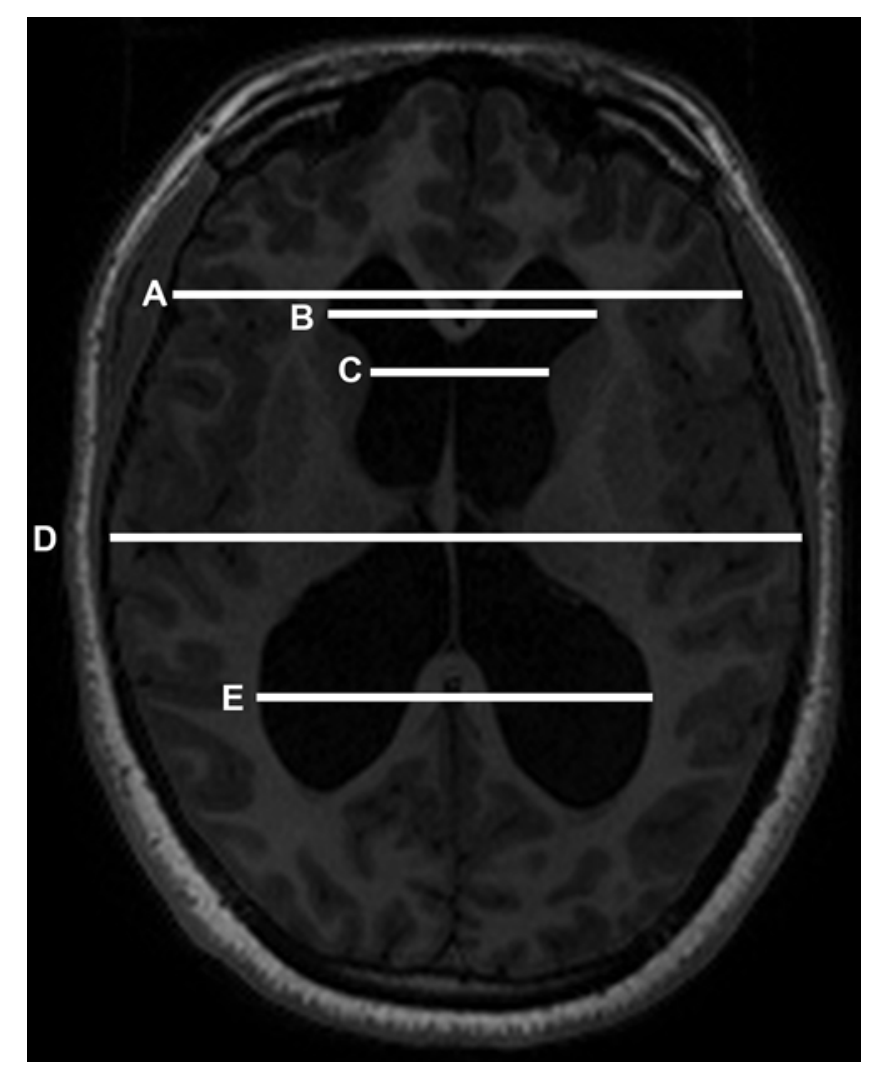

FIG. 1. Schematic displaying the measurements used in the linear indices. $F O H R=(B+E) / 2 D$; Evans' index $=B / A, F H R=B / D$; bicaudate index $=\mathrm{C} / \mathrm{A}$. tifact that precluded volume calculation. Volume and linear indices were determined independently by separate operators who were blinded to each other's results. A total of 22 subjects with hydrocephalus and 21 controls were included.

Reproducibility of the linear indices was evaluated in the hydrocephalus subjects and a subset of 14 control subjects. A second rater determined the linear indices in these subjects. The concordance correlation coefficient between the 2 raters was calculated.

The fraction of the brain volume occupied by the ventricles was estimated in 2 ways: first, by dividing ventricular volume by the ICV delineated as previously described. Second, the ventricle-to-brain volume ratio was estimated by dividing the ventricular volume by an estimated brain volume. This estimation of brain volume was based on the measured maximum dimensions of the brain, excluding the cerebellum. The volume of a half-ellipsoid with these dimensions was calculated as an approximation of the brain volume (ABV) according to the formula $1 / 3 \pi x y z=$ $\mathrm{xyz}$, where $\mathrm{x}, \mathrm{y}$, and $\mathrm{z}$, are the left/right, anterior/posterior, and superior/inferior dimensions of the brain, respectively (see Fig. 2).

Spearman's correlation coefficient was determined between both estimates of fractional ventricular volume and each of the linear indices as well as head circumference. The linear indices were also compared with absolute ventricular volume. Steiger's z-test was used to test for significant differences between the correlation coefficients? Bonferroni correction was used to correct for multiple comparisons.

The clinical parameter head circumference was also compared with ICV, ventricular volume, and fractional ventricular volume. Head circumference was considered both as an absolute measure and as a z-score that was corrected for age.

Additionally, brain volumes were compared between the 2 groups. Brain tissue was estimated by subtracting the ventricular volume from the ICV. A nonlinear monoexponential model was fit to the nonventricular brain volume. The residual volume after removing the age effect was then used to compare patients with hydrocephalus to

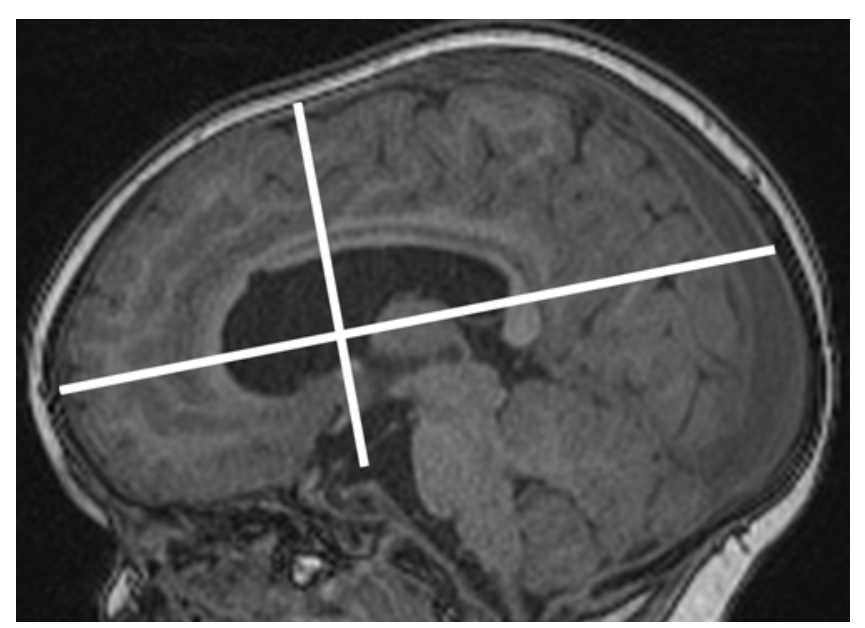

FIG. 2. The anteroposterior and superoinferior dimensions of the brain used to calculate the ABV are shown. 
controls using a t-test. All calculations were performed using MATLAB (MathWorks).

\section{Results}

All of the linear indices displayed a significant correlation $(\mathrm{p}<0.05)$ with the ventricular ICV-ratio (Fig. 3A, D, and $\mathrm{G}$ ) and $\mathrm{ABV}$ (Fig. 3B, E, H, and K), and the ventricular volume (Fig. 3C, F, I, and L). FOHR displayed a stronger relationship than the other indices with respect to all 3 volume measures (Fig. 3, compare the top row with the other rows of indices; $p<0.0001)$. Reproducibility of linear indices was very good; the correlation coefficient for
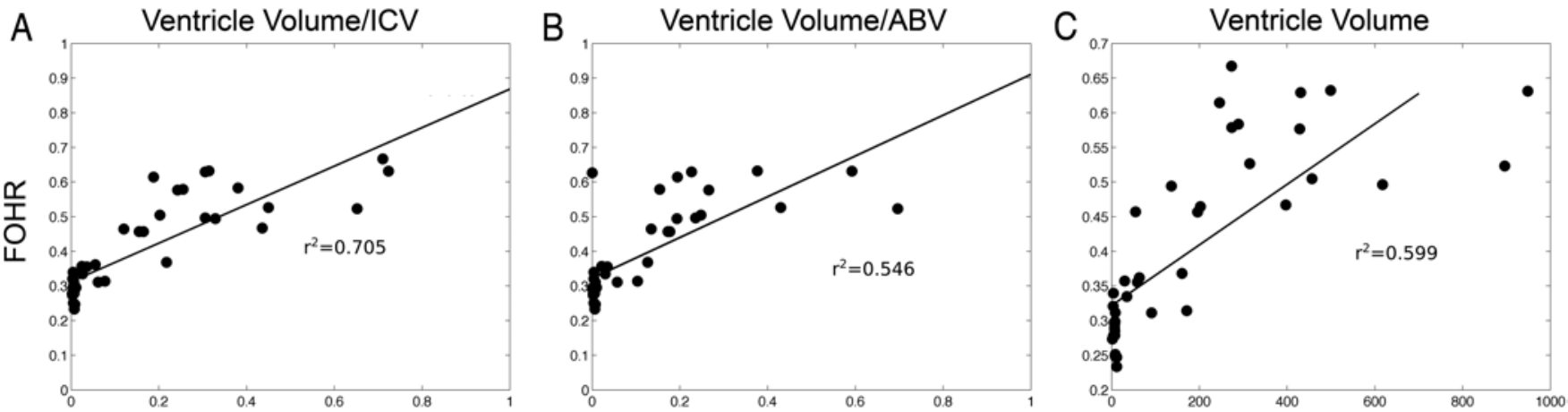

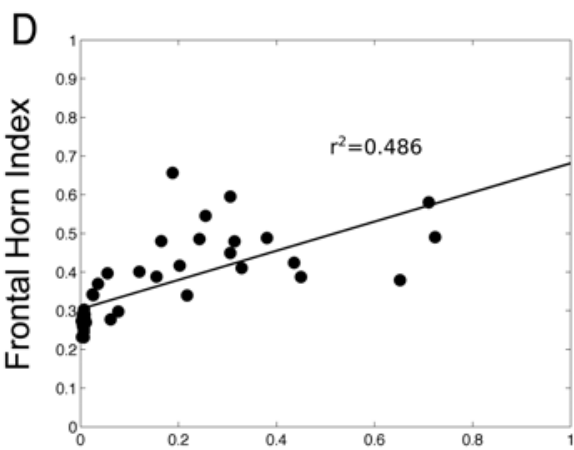

$\mathrm{H}$
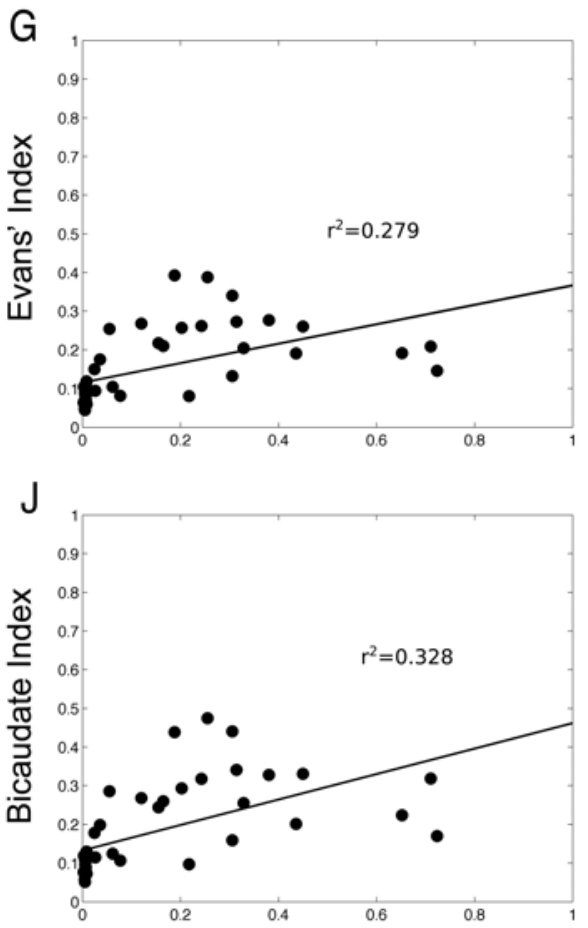

E
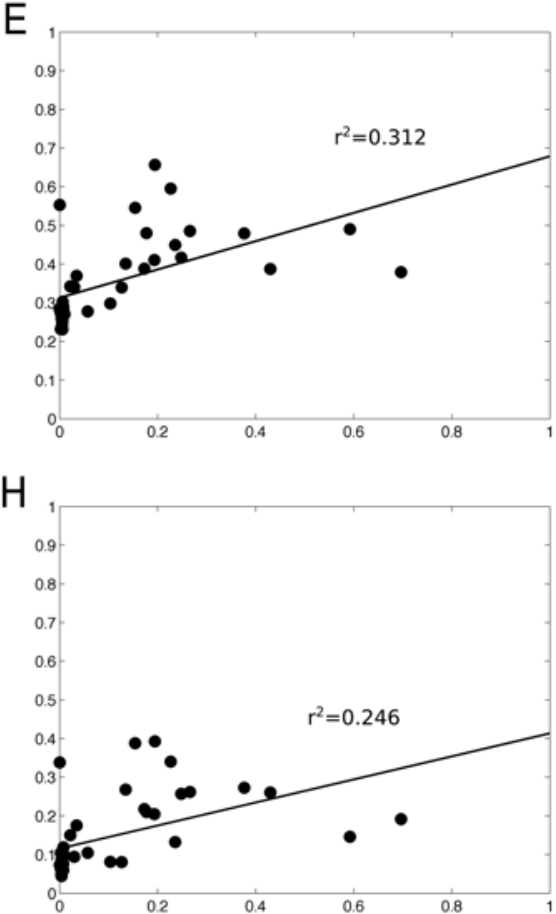

$\mathrm{F}$
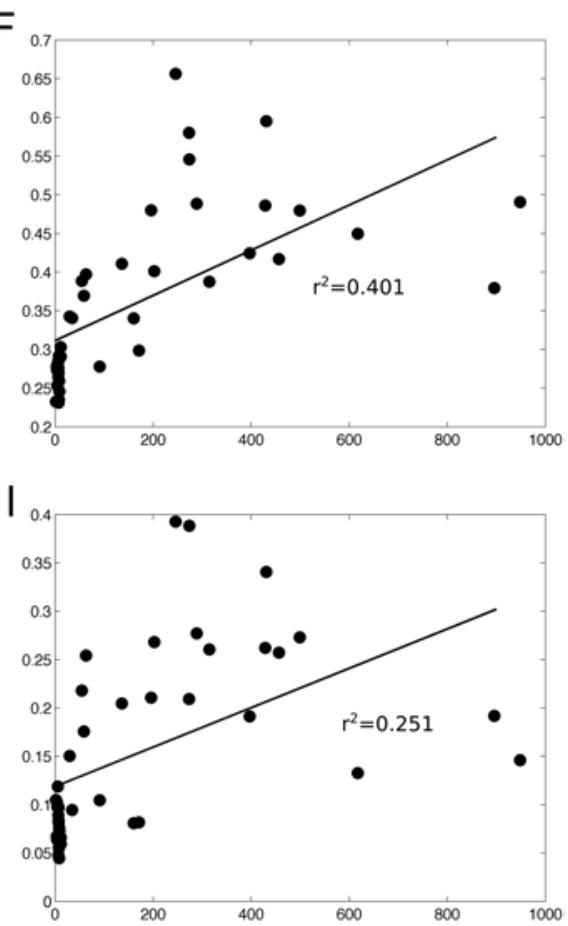

$\mathrm{K}$

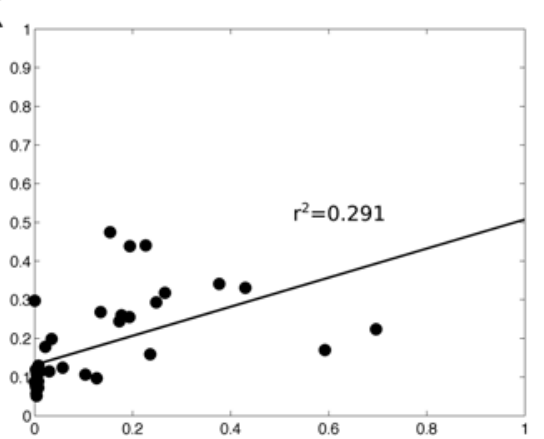

$\mathrm{L}$

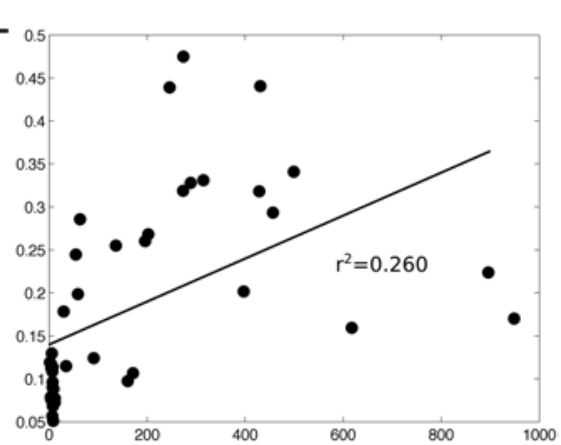

FIG. 3. The relationships between the linear indices and the 3 ventricular volume measures are shown. FOHR consistently provides the greatest correlation coefficient $(A-C)$ compared with the frontal horn index (D-F), Evans' index (G-I), or the bicaudate index $(\mathrm{J}-\mathrm{L})$. All coefficients are statistically significant $(p<0.05)$. 
the FOHR was 0.86 , for Evans' index it was 0.83 , for the frontal horn index it was 0.90 , and for the bicaudate index it was 0.81 . Intrarater reproducibility measures were slightly higher: the correlation coefficient for FOHR was 0.92 , for the frontal horn index it was 0.93, for Evans' index it was 0.88 , and for the bicaudate index it was 0.82 .

Head circumference was only very weakly associated with ventricular volume (Fig. 4A). However, it did display a strong correlation with ICV (Fig. 4B). This leads to children with larger circumferences having lower fractional ventricular volumes (Fig. 4C). Normalization of head circumference did not produce a correlate of ventricular volume (Fig. 4D).

Brain tissue volume was not found to be reduced in subjects with hydrocephalus compared with controls (Fig. $5 ; \mathrm{p}=0.30$ ).

\section{Discussion}

Linear indices of ventricular size have been used in patients with hydrocephalus since before tomographic imaging systems made volume measurements a viable possibility, and they are still the dominant method of evaluating ventricular size.

We found that FOHR had the strongest relationship with actual ventricular size, although other ventricular size indices also had statistically significant associations. This is consistent with prior studies by O'Hayon et al. ${ }^{8}$ and
Jamous et al. ${ }^{5}$ Jamous et al. suggested that FOHR was inaccurate in subjects with asymmetrical or slit ventricles. Our population only included 5 subjects with asymmetrical ventricles and none with slit ventricles according to the criteria used by Jamous et al., which precludes us from evaluating FOHR in these circumstances. Despite the different imaging modality used, our results are also consistent with those of O'Hayon et al., who found that FOHR was the most accurate modality considered, with others, such as Evans' index and the bicaudate index, being less accurate. ${ }^{8}$ We do expand upon these prior efforts in 2 ways. First, we measured relative ventricular size using the entire ventricular and intracranial volumes, unlike O'Hayon et al., who used a single slice. Second, we performed statistical comparisons of the various linear measures to compare them directly, instead of simply presenting point estimates of the correlation coefficients.

One potential confounding factor is the presence of enlarged subarachnoid spaces in children with hydrocephalus, which will result in the ICV not reflecting the actual amount of brain parenchyma. Accurate measurement of the subarachnoid volume is very difficult due to its complex structure and poor contrast. To overcome this issue, we developed the ABV, which depends only on the size of the brain parenchyma. The harmony of comparisons using either ICV or ABV indicates that the conclusions are not dependent on the volume of the subarachnoid spaces.

In general, if absolute ventricular volumes are needed,
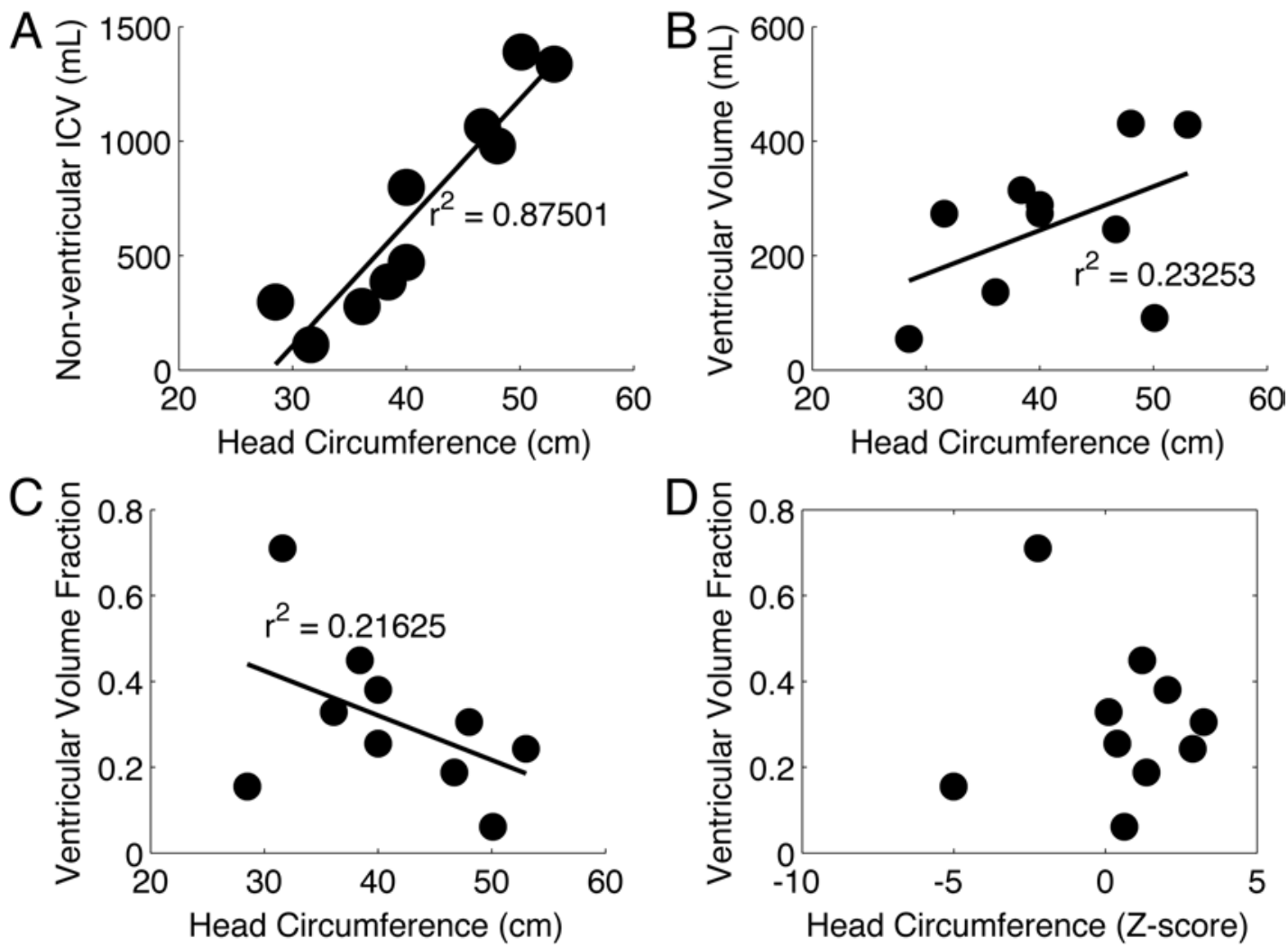

FIG. 4. The relationship between head circumference and ICV (A), ventricular volume (B), relative ventricular volume (C), and relative ventricular volume when head circumference is expressed as a z-score (D) is shown. Head circumference in hydrocephalus appears to be more influenced by ICV than by ventricular volume. 


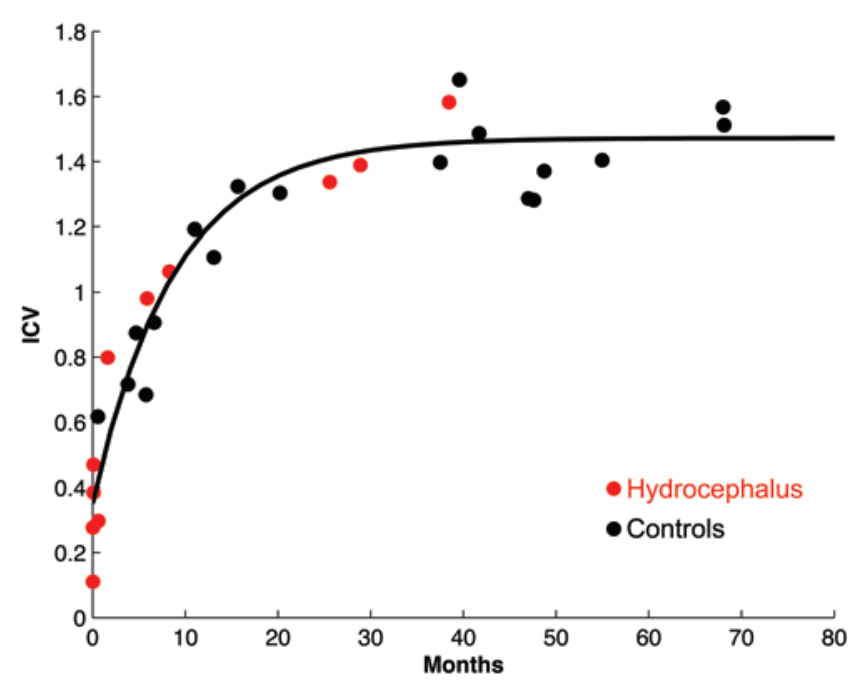

FIG. 5. Nonventricular brain volume is shown as a function of age in controls and children with hydrocephalus. The 2 plots are not significantly different, suggesting that ventricular enlargement does not compromise growth of brain tissue in patients with congenital hydrocephalus. Figure is available in color online only.

the use of absolute measurements is preferred to normalized ones. FOHR, however, was a good predictor of both absolute and relative ventricular volume. Other indices are still associated with ventricular size and may be appropriate clinically.

The primary limitation regarding the accuracy of our measurements is that we were not able to separate CSF in the subarachnoid space from the rest of the ICV because of the complex geometry of CSF in the sulci. To compensate for this, we performed 2 independent normalizations of the ventricular volume using both the ICV and ABV. Since the 2 strategies produced concordant results, we do not believe that subarachnoid space significantly alters our conclusions.

Perhaps surprisingly, the decrease in residual ICV due to ventricular enlargement was not detectable in this study. This suggests that, at least in this cohort of young subjects with congenital hydrocephalus, the increase in ICV secondary to hydrocephalus may preserve total brain volume.

\section{References}

1. Apostolova LG, Green AE, Babakchanian S, Hwang KS, Chou YY, Toga AW, et al: Hippocampal atrophy and ventricular enlargement in normal aging, mild cognitive impairment (MCI), and Alzheimer Disease. Alzheimer Dis Assoc Disord 26:17-27, 2012
2. Barr AN, Heinze WJ, Dobben GD, Valvassori GE, Sugar O: Bicaudate index in computerized tomography of Huntington disease and cerebral atrophy. Neurology 28:1196-1200, 1978

3. Bourne SK, Conrad A, Neimat JS, Davis TL: Linear measurements of the cerebral ventricles are correlated with adult ventricular volume. J Clin Neurosci 20:763-764, 2013

4. Hobbs NZ, Barnes J, Frost C, Henley SM, Wild EJ, Macdonald $\mathrm{K}$, et al: Onset and progression of pathologic atrophy in Huntington disease: a longitudinal MR imaging study. AJNR Am J Neuroradiol 31:1036-1041, 2010

5. Jamous M, Sood S, Kumar R, Ham S: Frontal and occipital horn width ratio for the evaluation of small and asymmetrical ventricles. Pediatr Neurosurg 39:17-21, 2003

6. Meese W, Kluge W, Grumme T, Hopfenmüller W: CT evaluation of the CSF spaces of healthy persons. Neuroradiology 19:131-136, 1980

7. Meng XL, Rosenthal R, Rubin DB: Comparing correlated correlation coefficients. Psychol Bull 111:172-175, 1992

8. O'Hayon BB, Drake JM, Ossip MG, Tuli S, Clarke M: Frontal and occipital horn ratio: a linear estimate of ventricular size for multiple imaging modalities in pediatric hydrocephalus. Pediatr Neurosurg 29:245-249, 1998

9. Synek V, Reuben JR, Du Boulay GH: Comparing Evans' index and computerized axial tomography in assessing relationship of ventricular size to brain size. Neurology 26:231233, 1976

10. Tate DF, Khedraki R, Neeley ES, Ryser DK, Bigler ED: Cerebral volume loss, cognitive deficit, and neuropsychological performance: comparative measures of brain atrophy: II. Traumatic brain injury. J Int Neuropsychol Soc 17:308-316, 2011

11. Yuan W, Holland SK, Shimony J, Jones BV, Mangano FT, Limbrick D, et al: Quality Assurance in Multi-Institution and Multi-Scanner MRI Neuroimaging Research. (http:// www.ipr2011.org/documents/536.pdf) [Accessed January 15, 2015]

\section{Author Contributions}

Conception and design: Ragan, Jones, Mangano, Yuan, Limbrick. Acquisition of data: Ragan, Cerqua, Nash, Yuan. Analysis and interpretation of data: Ragan, McKinstry, Shimony, Limbrick. Drafting the article: Ragan. Critically revising the article: Ragan, Cerqua, McKinstry, Shimony, Jones, Mangano, Holland, Yuan, Limbrick. Reviewed submitted version of manuscript: Ragan, Cerqua, McKinstry, Shimony, Jones, Mangano, Holland, Yuan, Limbrick. Approved the final version of the manuscript on behalf of all authors: Ragan. Statistical analysis: Ragan. Administrative/ technical/material support: Yuan, Limbrick. Study supervision: Limbrick.

\section{Correspondence}

Dustin K. Ragan, 660 S. Euclid, Campus Box 8116, St. Louis, MO 63110. email: ragand@neuro.wustl.edu. 